
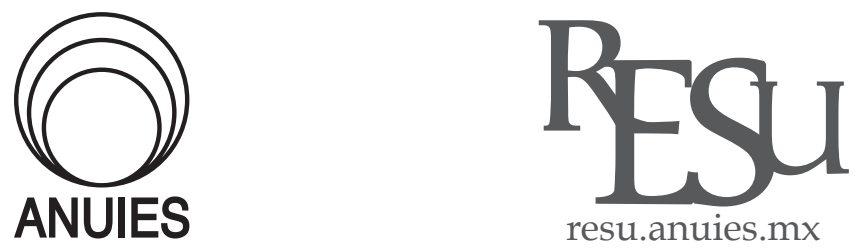

REVITA DEL
EDICACIÓN
SUPERIOR

ARTÍ́CULO

\title{
Oportunidades de acceso a la educación superior y al trabajo profesional. Un estudio de caso*
}

\author{
Student Dropout in Technical-Professional Higher Education: Exploring \\ factors that influence freshmen students

\section{Guadalupe García**} \\ * Un breve avance de los resultados de esta investigación fue presentado como ponencia en el XIV Congreso \\ Nacional de Investigación Educativa de 2017. \\ ** Departamento de Sociología. Universidad Autónoma Metropolitana-Unidad Azcapotzalco \\ Correo electrónico: gcgpe@correo.azc.uam.mx
}

Recibido el 26 de octubre del 2017; aceptado el 28 de enero del 2019.

\section{Resumen}

Este artículo analiza la influencia de características sociodemográficas, académicas y laborales en la desigualdad de oportunidades. Es un estudio de caso sobre condiciones y trayectorias académicas de los alumnos inscritos en la Universidad Autónoma Metropolitana entre 1990 y 2005; y para un grupo de sus egresados, se considera la relación entre factores académicos y condiciones laborales. Nos basamos en el modelo analítico de Bartolucci, quien a partir de Schütz plantea que los universitarios toman decisiones orientadas por la valoración que hacen de sus condiciones, desde su particular situación biográfica e histórica, y como parte de un grupo social específico.

Palabras clave: Educación Superior; Oportunidades educativas; Oportunidades laborales; Trayectoria escolar; Egresados. 


\begin{abstract}
This article discusses the influence of socio-demographic, academic and job characteristics in the inequality of opportunities. It is a case study on conditions and academic trajectories of the students enrolled in the Universidad Autónoma Metropolitana between 1990 and 2005; and for a group of graduates, is considered the relationship between academic factors and job conditions. The research uses the analytical model of Bartolucci, who inspired on Schütz, proposes that students make decisions guided by the assessment they make, based on their conditions, from their particular biographical and historical situation, and as a part of a specific social group.
\end{abstract}

Keywords: Higher Education; Educational opportunities; Job opportunities; School trajectories; Graduates.

\title{
Objetivos y fundamentación
}

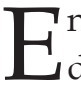

n esta investigación analizamos la posible influencia en la desigualdad de oportunidades educativas y los logros, en el mundo del trabajo de algunas características sociodemográficas, académicas y laborales, en un grupo de alumnos y exalumnos de la Universidad Autónoma Metropolitana.

Partimos del supuesto de que en tales características y condiciones de los sujetos, por el significado social que tienen, es posible identificar orientaciones que incidirán en la toma de decisiones sobre su escolaridad y su inserción al mercado laboral. Este planteamiento tiene sustento en la teoría sociológica de Alfred Schütz, quien establece que los actores definen sus posibilidades de acción orientados por la interpretación que cada uno hace de sus condiciones, experimentadas desde la particular situación biográfica; desde la experiencia sedimentada a través del tiempo de vida, alimentada en términos de significados por antecesores y contemporáneos (Schütz, 1962).

También se fundamentó nuestro trabajo en investigaciones clásicas que sobre las oportunidades educativas se han hecho en el mundo (Bernstein, 1989; Boudon, 1983; Bourdieu y Passeron, 1973; Bowles y Gintis, 1983; Coleman et al., 1966) y en México (Bartolucci, 2000; Guzman, 1994; Guzman y Serrano, 2011; Rodriguez Gómez, 1996; Silva Laya, 2014), que han dado importancia a características y condiciones tales como origen social familiar de los estudiantes, sexo, edad, estado civil, desempeño académico, y condiciones de inserción a la actividad laboral para explicar el resultado diferencial del logro. 
Particularmente el enfoque que adoptamos se desprende de la propuesta analítica de Bartolucci, quien con base en Weber y en Schütz establece que la explicación del comportamiento social implica la comprensión de los motivos y significaciones que los sujetos atribuyen a sus actos; y asume que las características que distinguen a los integrantes de una generación estudiantil, en cierto momento de su trayectoria, dan cuenta del entrecruzamiento de acciones que fueron realizando en circunstancias específicas, sustentadas en las cualidades y los atributos que orientan sus decisiones. Aquí la extracción social, la edad, el género o el éxito en los estudios aparecen como indicadores del sentido que pudiera tener para los estudiantes, su posición y sus circunstancias sociales específicas (Bartolucci, 2000).

Aunque existe una amplia bibliografía al respecto, es relevante volver sobre el tema en la medida en que podemos observar la forma que van adoptando a través del tiempo las condiciones de estas relaciones. Hacer un estudio de caso como el presente permite tener conocimiento de ciertos componentes que incidieron en las condiciones de oportunidad educativa para el amplio grupo de jóvenes que cursaron estudios en la UAM en la década de los noventa y los inicios del siglo XXI (1990-2005). Así mismo, brinda información más detallada sobre un grupo más pequeño (7,450 casos), compuesto por exalumnos egresados de esta Universidad que fueron encuestados por la propia institución sobre sus trayectorias y condiciones laborales, - algunos en 2006 (cohortes de egreso 1998 y 2003) y otros en 2012 (cohortes de egreso 2005 y 2008 - en los Estudios de Seguimientos de Egresados de los que se nutre la presente investigación. También ese cuestionario nos permitió conocer la escolaridad de los padres que es un dato frecuentemente utilizado en la investigación social como indicador del nivel socioeconómico de la familia de origen.

Sobre el grupo más amplio al que aludimos primero, compuesto de 160,445 casos, contamos solamente con datos sociodemográficos y de carácter académico captados por la oficina de Sistemas Escolares relativos a sus estudios universitarios y concentrados en el Archivo General de Alumnos de la UAM (AGA), base de datos que se alimenta con los resultados de cada trimestre escolar. De ahí obtuvimos información sobre edad, género, estado civil de los alumnos, así como sobre su promedio de egreso del bachillerato, puntaje en el examen de admisión, trayectorias educativas en la licenciatura y promedio de calificaciones al terminar los estudios. La observación sobre este amplio grupo se enfocó a las oportunidades educativas. Para el análisis del grupo restringido, el de los egresados, fue posible integrar las bases de la información académica del AGA y la laboral generada por los Estudios de Se- 
guimiento de Egresados permitiéndonos observar la relación entre factores sociales, académicos y laborales en cada uno de los casos.

Analizar las condiciones de oportunidad para este amplio grupo de jóvenes de una de las instituciones de educación superior más importantes de la Zona Metropolitana de la Ciudad de México, a lo largo del periodo señalado, nos permite aportar una pieza significativa a la comprensión del fenómeno, en un momento particular de la historia en nuestro país.

El problema del que parte la presente investigación surge de observar que, no obstante que la ampliación de la oferta educativa impulsada por el Estado mexicano desde finales de los años 50 sigue siendo ventajosa para los grupos sociales mejor posicionados, a partir de los años 70 es notable un cambio en la composición social del alumnado en las universidades públicas mexicanas, incrementándose la proporción de jóvenes procedentes de familias de sectores económicos medios y bajos (Bartolucci, 1994; Covo, 1995; Guzmán y Serrano, 2007; Rodriguez Gómez, 1996). La pregunta que nos planteamos entonces fue: ¿quiénes son los jóvenes que lograron beneficiarse de las oportunidades educativas?, y de manera más concreta, ¿cuáles de sus características pueden haber incidido en sus trayectorias y en sus logros? ¿Ser un buen alumno significa ventajas en las futuras condiciones laborales? ¿Tiene peso alguno la trayectoria laboral previa? Para dar respuesta a estas interrogantes nos propusimos realizar el estudio de un caso que fuera significativo, eligiendo para nuestra observación a alumnos y exalumnos de la UAM, por ser ésta una institución pública altamente valorada por los aspirantes a la educación superior en la Zona Metropolitana de la Ciudad de México. Prueba de ello es que en 2015 se presentaron más de 90,000 jóvenes al proceso de selección (UAM, 2015).

Empezamos por establecer el contexto en el que se desenvolvieron las trayectorias escolares y laborales de los jóvenes que analizamos, porque es el escenario en el que se generaron sus proyectos y el marco que delimitó la plausibilidad de sus oportunidades. Establecido el escenario, procedimos a analizar las características de los jóvenes universitrios buscando evaluar el peso de éstas en sus logros educativos y laborales. Empezamos por observar la posible incidencia del nivel de estudio de los padres como indicador del nivel social familiar de origen en su desempeño académico y en el logro laboral, en distintos momentos de estos trayectos. Luego quisimos dimensionar el peso de los resultados académicos en el nivel de éxito alcanzado en el trabajo, suponiendo que un mejor alumno podría lograr una situación más ventajosa. Enseguida, evaluamos el peso que la trayectoria laboral previa podía tener hacia el futuro; y por último analizamos las diferencias que se generaron en los anteriores cortes analíticos, para los hombres y mujeres. 
Como ya lo señalamos previamente, el análisis relativo a las condiciones laborales sólo fue posible para el grupo de los egresados. Para todos los demás, sólo hacemos referencia a la relación entre sus características y sus logros académicos. Para realizar estas observaciones utilizamos el paquete estadístico sPSs con el cual elaboramos tablas de contingencia y medidas de dispersión, y comprobamos grados de asociación de las variables seleccionadas, con la prueba estadística Chi-cuadrada.

\section{El Contexto}

De manera muy breve señalemos que el contexto de oportunidad educativa durante el periodo en el que ingresó la mayor parte de los jóvenes, objeto de este estudio (1990-2000), se caracterizó por un importante crecimiento de los egresados de la educación media superior en un 32\%, impulsado no sólo por el crecimiento demográfico del grupo en edad sino también por un cambio en las expectativas educativas de las mujeres, y por un crecimiento insuficiente de la oferta de educación superior pública para atender a este incremento de la demanda potencial (Álvarez Mendiola, 2011), como consecuencia de la crisis económica que desde entonces se hace sentir en nuestro país, y del cambio en la política pública estatal que decidió restringir sus gastos.

Esta situación estimuló el crecimiento de instituciones de educación superior privadas, las cuales se hicieron cargo de cubrir una parte de la demanda: fundamentalmente la de aquellos que no encontraron lugar en las instituciones públicas y estuvieron en condiciones de poder cubrir los gastos correspondientes (Acosta, 2005; De Garay, 2011). Para quienes no tenían tal posibilidad, implicó la reducción de oportunidades de acceso a la educación superior, constatable en el creciente número de no aceptados. En la UAM, por ejemplo, el número de aspirantes se duplicó en el periodo, pasando de 26,677 a 59,764, mientras que el número de admitidos se mantuvo prácticamente igual: 11,838 en 1990 y 12,364 en 2005 (UAM, 2011).

En cuanto al contexto laboral para los profesionistas en los primeros años del siglo XXI, lo que se ha observado es que existe una tendencia a la disminución de las ventajas laborales que en el pasado tuvieron los egresados de la educación superior. No obstante que este grupo tuvo en general mejores condiciones de trabajo que quienes tenían niveles menores de estudios, vimos que en los últimos 20 años se ha ido reduciendo su acceso a puestos de mando, que han ido realizando con más frecuencia tareas que antes hacían personas con niveles de estudios más bajos, y que en menor proporción alcanzaron los ingresos altos que beneficiaron a los egresados de las universi- 
dades en los años 70 u 80 (ANuies, 2003, 2012; E. De la Garza y Gaspar, 2009; M. De la Garza, 2015; Hernández Laos, 2004; Hernández Laos y al, 2012). (ANuies, 2003, 2012; E. De la Garza y Gaspar, 2009; M. De la Garza, 2015). Estos fenómenos se explican más que por las características de los egresados de estudios superiores, por el tipo de desarrollo económico y la consecuente falta de crecimiento o incluso el decrecimiento de puestos de mando o de empleos de profesionistas específicamente, agravado por la disminución del empleo por parte del gobierno, en el marco de las políticas de restricción del gasto público que tomó fuerza en los 90. Agreguemos una nota más en relación con las condiciones de la Zona Metropolitana de la Ciudad de México. A diferencia de lo que ocurre en otras regiones del país, en el valle de México había, al inicio del siglo, proporcionalmente más profesionistas compitiendo por cada puesto que requería estudios de nivel superior (ANUIES, 2003). No obstante, estos egresados ocuparon puestos como personal directivo más frecuentemente que el promedio nacional (7.9\% contra 5.9 respectivamente) y trabajaron como profesionales también más habitualmente $(28.8 \%$ contra 22.5). Esto es comprensible dada la concentración extraordinaria de bienes y servicios de esta megalópolis, conformada a lo largo de una historia de desarrollo económico, político y social fuertemente centralizados. En la ZMCM, ha habido condiciones menos desfavorables en términos de oportunidades educativas y también en el ámbito del trabajo.

\section{¿Oportunidad para quién?}

El resultado del análisis estadístico nos mostró que el origen social marca diferencias en las oportunidades educativas de manera muy clara. Coincidiendo con lo encontrado por Bartolucci (1994), Rodríguez (1996) y por Guzmán y Serrano (2011) en sus estudio sobre el proceso de selección en la UNAM, el nivel de estudios de los padres de los jóvenes que tuvieron éxito en ingresar a la UAM fue mayor que el de los no aceptados. En 1990, dos de cada tres, entre los elegidos, tenían padres que habían realizado estudios más allá de la secundaria, mientras que, entre quienes no tuvieron éxito, sólo uno de cada cuatro estaba en tal condición (López Zárate, González Cuevas, y Casillas Alvarado, 2000). En 2005 la brecha se redujo, pero se mantuvo la condición ventajosa para quienes procedían de familias con mayores estudios: las cifras fueron de 50.5 y $41 \%$, respectivamente. Entre los aceptados, los padres con estudios de educación superior eran un 10\% más frecuentes que entre los no aceptados, quienes por su parte tenían $10 \%$ más en los rangos relativos a educación primaria y secundaria como máximo. Consecuentemente, con lo anterior en- 
contramos también que tuvieron mayores posibilidades de ser seleccionados quienes provenían de instituciones privadas, hecho que ha sido observado en otros estudios sobre el ingreso a la UAM (De Garay y Sánchez, 2012).

Sin embargo, la magnitud de la demanda de instituciones privadas es menor que la de las instituciones públicas, y la proporción de quienes eran hijos de padres con niveles educativos bajos no fue pequeña. Así es que la población que ingresó a esta institución en la década de los noventa y principios del siglo XXI se caracterizó por ser un grupo de hombres y mujeres heterogéneo en términos socioeconómicos, que ha logrado abrir para sí las puertas de la universidad gracias al carácter cuasi gratuito de esta institución, así como al mecanismo de admisión que en el periodo aquí estudiado establecía el corte entre aceptados y no aceptados con base en el mérito en el examen de selección y en función del cupo o capacidad de atención definida por la institución para cada carrera, en cada uno de los planteles o Unidades Académicas. El análisis de los datos nos mostró que la oportunidad de ingreso a la UAM ha estado asociada a ser jóvenes de alrededor de 20 años de edad, haber tenido una trayectoria escolar relativamente continua hasta haber concluido el bachillerato y no haber adquirido compromisos conyugales o de paternidad antes de postular. El carácter diverso de la composición social del alumnado es una característica común en las instituciones de educación públicas del país, producto del proceso de masificación iniciado en la segunda mitad del siglo xx, documentada desde hace ya mucho tiempo (Fuentes, 1983).

\section{El trayecto en la universidad}

Una vez pasado el filtro de la admisión, en el análisis de la trayectoria en los estudios universitarios encontramos que el factor socioeconómico perdió peso ante el del mérito académico. Las posibilidades de dejar los estudios o de llevarlos a término en la UAM no dejan de estar asociados al nivel sociocultural de la familia. Pero como lo señalara ya Bartolucci en relación con los alumnos de la UNAM (Bartolucci, 2000), no es suficiente este factor para explicar el resultado académico del grueso de la población analizada. Como veremos más adelante, muchos jóvenes, cuyos padres tuvieron baja escolaridad y que ingresaron a la vida laboral mientras estudiaban (esto último sólo lo pudimos establecer para el grupo restringido, el de los egresados que analizamos con mayor profundidad), fueron buenos y muy buenos estudiantes y concluyeron sus licenciaturas. Hablemos antes de aquellos que fueron dejando esta opción educativa sin concluir, entre quienes ingresaron a la UAM en la década de los noventa y principios del siglo XXI. 


\section{Los que no concluyeron}

Del total de alumnos que ingresaron a la UAM entre 1990 y 2005, 4 de cada diez no permanecieron hasta terminar sus estudios. En este trabajo no contamos con información que nos permita saber más que algunos datos escolares al respecto. A través de éstos tenemos conocimiento de que a lo largo del primer trimestre uno de cada tres había dejado la institución (32.5\% para ser exactos) y que $15 \%$ lo hizo en los dos siguientes trimestres. Esto es, antes de iniciarse el segundo año escolar, casi la mitad de los alumnos que no concluirán, han ya abandonado los estudios. A partir de entonces, el ritmo de pérdida de alumnos se desacelera gradualmente. Si hacemos esta observación por cohorte de ingreso, vemos que, a partir del año 2000, hay una incremento en la proporción del abandono particularmente en el primer trimestre. No sabemos realmente si fueron alumnos, o si muy probablemente sean aquellos que buscaron una opción en otra institución, y que implica que realmente no ingresaron en la UAM.

Algunos investigadores han hecho ver que el problema de dejar inconclusos los estudios en las instituciones de nivel superior debe ser tratado con cuidado, ya que en los datos institucionales frecuentemente es indistinguible la situación de quienes han abandonado definitivamente los estudios, de la de quienes migran hacia otras universidades (Wietse De Vries, 2011; Sánchez Medina, 2010; Tinto, 1989). Tanto en uno como en otro casos, el abandono escolar se produce con mayor frecuencia durante el primer año. Una gran cantidad de aspirantes hacen los trámites para ingresar a distintas universidades con el fin de ampliar sus probabilidades de tener algún lugar seguro. Habiendo sido aceptados en más de una institución, la elección de una implica renunciar en otra; en este caso, el problema tiene sobre todo un carácter administrativo. También lo es cuando los alumnos se sienten insatisfechos con el programa o la universidad a que han ingresado y se salen en búsqueda de una opción más adecuada a sus expectativas. ${ }^{1}$

Pero el abandono que preocupa más socialmente es el que implica dejar definitivamente los estudios universitarios: alguien para quien la oportunidad pareció posible, pero que no logró consolidarla. El problema ya no es sólo en términos de eficiencia institucional y de administración y "dispendio" de los recursos. Cuando los alumnos interrumpen definitivamente la universidad, estamos ante un problema de carácter social. Vincent Tinto (1989), a principios de los noventas, señaló la necesidad de diferenciar entre

\footnotetext{
${ }^{1}$ En las universidades mexicanas durante el primer año de estudios se pierde entre el $25 \%$ y $35 \%$ de los recién matriculados (De Garay, 2006).
} 
estos dos tipos de abandono: el de quien cambia porque tiene o busca una mejor opción, y el de quien pierde la oportunidad de continuar su formación hasta terminar una carrera.

Para este segundo tipo de abandono, Tinto generó un modelo explicativo basado en la teoría de la solidaridad o de la integración de Durkheim: a mayor sentido de pertenencia, menor probabilidad de "suicidio académico". Alumnos que se sienten integrados a la institución, ya sea por factores académicos o bien de carácter social, es menos probable que dejen inconclusa la carrera.

El problema del abandono escolar no es privativo de la UAM ni del país, pero en México alcanza proporciones más altas que en los países desarrollados, llegando a $42 \%$, mientras que en los países miembros de la OECD sólo tres de cada 10 alumnos de la educación superior dejan sus estudios inconclusos (OCDE, 2010).

Adrián de Garay ha planteado que el ingreso a la universidad implica la inserción en un ambiente que los alumnos perciben como diferente, con cambios fuertes en términos culturales y sociales, al cual deberán adaptarse. Según este autor, uno de los motivos más poderosos del abandono masivo durante el primer año en la educación superior en México se debe a que no se ha producido la adecuación necesarias entre las nuevas exigencias intelectuales y de prácticas de estudio y de trabajo en la universidad y las formas previas con que llegan los alumnos. El problema sería la incapacidad para detectar, descifrar e incorporar los códigos de afiliación (De Garay, 2001, p. 88).

Aunque sin duda es relevante esta observación, el problema del abandono no se reduce a una falta de destrezas académicas o de inserción social o sentido de pertenencia en términos de V. Tino. Dejando a un lado a quienes simplemente optaron por otra alternativa y por ello dejaron el lugar ganado en un institución, que son parte del abultamiento del abandono temprano, podríamos señalar también exigencias extraescolares de carácter social como factores de peso que dan forma al problema. Por ejemplo, este proceso ocurre entre muchachos de todas las edades en lapsos similares, pero crece constantemente en relación con la edad. Mientras que entre aquellos que ingresaron teniendo 19 el abandono implicó a la mitad, (49.2\%), entre los que habían cumplido ya 24 años contabilizó a más de 2 de cada 3 (67.7\%). Patricia Muñiz (1997) al analizar las características de dos generaciones de alumnos correspondientes a 1979 y 1987 observa que el haberse incorporado al trabajo, además de haber contraído compromisos conyugales (dos transiciones a la vida adulta), es un factor que incrementa las posibilidades de deserción entre los alumnos de la UAM, particularmente para el sector masculino. García Castro (2007), en un estudio sobre alumnos que ingresaron a la UAM en otoño de 
1991 (Generación 91-O), llega a resultados similares. En esta población, compuesta por 3,791 alumnos (80\% de quienes ingresaron en otoño y $47 \%$ del total del ingreso anual), se observa cómo la incorporación a la vida laboral está directamente relacionada con el género y con la edad. La proporción de quienes trabajaban aumentó de $24 \%$ entre los que tenían 19 años o menos, a $57 \%$ entre los de 20 y 21, y a $82 \%$ en los más grandes. Así mismo fue posible establecer una relación entre la edad y el estado civil. Mientras que en los de 21 años o menos los casados sólo representaron 2.7\%, en los de más de 21, la proporción llegó a 20\% (García Castro y Bartolucci, 2007). En concordancia con lo expuesto por Muñiz, no es extraño que entre los más jóvenes (de 19 o menos) el abandono haya ocurrido entre uno de cada dos, mientras que en los mayores de 24 desertaran dos de cada tres.

El mismo De Garay, en una investigación posterior sobre alumnos de la UAM Azcapotzalco, generación de ingreso 2003, encuentra que es más frecuente entre aquellos alumnos que trabajaban mientras estudiaban, y entre quienes estaban casados o en unión libre. Aunque también identifica a un grupo que, más que tener problemas para continuar los estudios, parecen haber buscado una opción educativa diferente. Tal supuesto se desprende de que los muchachos de este grupo eran hijos de padres con los más altos niveles de educación, esto es, con educación superior. El trabajo de Bartolucci (2000) coincide también con estos resultados. Las nuevas responsabilidades personales limitan las posibilidades de seguir dando la misma prioridad a los estudios.

No tenemos información suficiente que nos permita abundar al respecto sobre la totalidad de los alumnos comprendidos en este estudio, pero sí sobre quienes ingresaron en Otoño de 1991.

Así pues, aunque no tenemos datos sobre su estado civil o la participación laboral del momento en que ingresó el resto de los alumnos incorporados durante todo el periodo que analizamos (1990-2005), podemos suponer que su edad nos da pista sobre la probabilidad de que hayan hecho tales transiciones mientras estudiaban la licenciatura y que pudieran ser un factor que incide en el resultado observado en el párrafo anterior.

En la investigación que elaboró Sánchez Medina sobre el abandono durante el primer año de estudio en alumnos de la UAM (2010), se establece que es mayor la deserción masculina que la femenina en general, coincidiendo con otras investigaciones en las que se muestra que es mayor la eficiencia terminal entre las mujeres que entre los hombres (Bartolucci, 2000; Mingo, 2007; Rodriguez Gómez, 1996). En la población que analizamos nosotros, la deserción implicó a dos hombres por cada mujer (65.3 de los desertores eran hombres, $34.7 \%$ de las mujeres). Entre las mujeres el abandono se llevó a cabo 
en menos tiempo, concentrándose un poco más en los primeros trimestres. Al año de haber ingresado, ya lo habían hecho la mitad de las mujeres de este grupo. Entre los hombres la cifra se ubica seis puntos por debajo.

Una condición última a la que podemos aludir es a la posible relación entre el promedio de calificaciones obtenidas en el bachillerato y su incidencia en el éxito o fracaso para concluir los estudios universitarios. A mayor promedio fue menor el abandono escolar en la UAM, tanto entre los hombres como entre las mujeres. Éste es un hecho que ha sido observado ya por investigadores (Sanchez Medina, 2010) y gestores en varias instituciones de educación superior y que ha implicado cambios en sus procedimientos de selección para la admisión. Tal es el caso en la UAM, donde a partir del 2008 (posterior al periodo que analizamos en esta investigación) se estableció que el promedio del bachillerato representaría el 30\% de la calificación con la que se define la aceptación de los candidatos. El otro $70 \%$ lo constituye la calificación del examen de selección.

Cuadro 1

Abandono escolar, por promedio en el Bachillerato en relación con el grupo de hombres o mujeres que ingresaron a la UAM entre 1990 y 2005

\begin{tabular}{lccc}
\hline Promedio en Bachillerato & Hombres & Mujeres & Total \\
\hline hasta 7.4 & 63.8 & 51.7 & 60.9 \\
\hline 7.5 a 7.9 & 60 & 46.9 & 55.4 \\
\hline 8 a 8.4 & 58.1 & 44.2 & 52.2 \\
\hline 8.5 a 8.9 & 54 & 41.4 & 47.7 \\
\hline 9 a 9.4 & 51.4 & 38.9 & 44.5 \\
\hline 9.5 y más & 47.1 & 36.3 & 40.6 \\
\hline
\end{tabular}

El problema del abandono se conforma pues de manera compleja y puede tardar mucho en consumarse. Dos años después de haber iniciado, 40\% de los alumnos que no concluirían sus estudios seguían intentándolo. Seis trimestres más tarde, cuando los más rápidos entre los compañeros de generación estaban egresando, quedaban aun, tratando de mantenerse dentro, uno de cada 5 entre los que no concluirían. A 1,980 alumnos, 1.4\% de quienes dejaron inconclusos sus estudios (del ingreso en la década de los 90 hasta 2005), les faltaron menos de 50 créditos para terminar (elaboración propia con base en el AGA de la UAM, actualizada en 2015).

Una observación sobre esta problemática a nivel de las Unidades Académicas de la UAM nos muestra que sin duda existe una incidencia significativa de este factor sobre la factibilidad de lograr concluir los estudios. Mientras 
que en la Unidad Iztapalapa sólo una tercera parte de los alumnos pudieron llegar a cubrir la totalidad de los créditos de sus carreras (35.5\%), en Xochimilco lo hicieron uno de cada dos (56.4\%), y en Azcapotzalco un poco menos de la mitad (40.4\%). Parte de la explicación sin duda tiene que ver con el modelo académico de cada una de estas Unidades. El sistema modular de Xochimilco es posible que favorezca un mejor desempeño en la medida en que genera un mayor sentido de integración de los alumnos, componente del cual ya hablamos como ventajoso con base en Tinto y De Garay, entre otros autores. Además, suponemos que en la medida en que en este sistema no se evalúan materias aisladas sino un módulo, una unidad que equivale a un número de créditos relativamente igual al de los que deberían ser cursados en un trimestre por los alumnos de las otras Unidades Académicas, ellos se enfrentan a aprobar o reprobar el conjunto. Es posible que esto genere una actitud de mayor esfuerzo ante la posibilidad de perderlo todo. Estos son algunos factores institucionales que se entretejen con las especificidades de los alumnos para dar forma a la trayectoria de abandono. En cuanto a estas últimas, probablemente el factor socioeconómico ha sido relevante, tal como ha sido señalado en las investigaciones sobre el tema (Rodriguez Gómez, 1996). No tenemos información específica sobre cada uno de estos alumnos, pero sabemos que los estudiantes de la Unidad Iztapalapa tienen un nivel socioeconómico más bajo que aquellos de las otras dos Unidades, y que el de Xochimilco es más alto.

Algunos autores han planteado que el enorme problema del abandono escolar demerita los supuestos avances en materia de democratización, y que la discriminación social persiste ya no como restricción de acceso educativo sino como fracaso en la posibilidad de mantenerse dentro del sistema y avanzar hasta la conclusión de los estudios, en gran parte cubierto bajo el manto legitimador de las políticas institucionales que establecen como criterios de selección capacidades e incapacidades académicas individuales sin ocuparse de garantizar condiciones mínimas para igualar oportunidades (De los Santos, 2004; Fernandez Mellizo-Soto, 2003; Granja, 1983). Aquí lo que observamos a partir de la información analizada es que el género, la edad, los momentos de transición a la vida adulta y los antecedentes escolares (factores que por su significación social inciden en la manera en la que cada individuo percibe sus posibilidades de acción), se entretejen en un momento histórico y con condiciones institucionales particulares, para dar mayor o menor viabilidad al proyecto educativo de cada uno. Ninguno de estos elementos por sí solo es determinante (el abandono en el nivel de la educación superior deja de estar ligado a la escolaridad de los padres de manera tan fuerte como en 
los ciclos escolares anteriores). Es el entramado particular de los distintos componentes el que alienta o no a los alumnos a seguir en el intento.

\section{Los que lo lograron}

De la totalidad de quienes ingresaron entre 1990 y 2005, el grupo de los alumnos que cubrieron exitosamente la totalidad de los créditos de su plan de estudios estuvo compuesto por 48,319 mujeres y 49,061 hombres. Fueron $60 \%$ del total de quienes ingresaron durante el periodo observado y en conjunto tuvieron calificaciones un poco más altas en el bachillerato que quienes abandonaron sus estudios en la UAM. El nivel de escolarización de los padres de estos egresados fue muy diverso, y como lo señalamos previamente, aunque sin duda incidió en la probabilidad de tener un mejor promedio académico, definitivamente no fue un factor determinante para ser un mejor alumno, tal y como puede apreciarse en la siguiente tabla relativa a los egresados comprendidos en esta investigación.

Cuadro 2

Índice de escolaridad de los padres, por promedio de calificaciones en la UAM

\begin{tabular}{|c|c|c|c|c|c|}
\hline \multirow[b]{2}{*}{ Índice de escolaridad de los padres } & \multicolumn{4}{|c|}{ Promedio final en la UAM } & \multirow[b]{2}{*}{ Total } \\
\hline & hasta 7.9 & de 8 a 8.4 & $\begin{array}{c}\text { de } 8.5 \text { a } \\
8.9\end{array}$ & 9 o más & \\
\hline Hasta primaria como máximo & $29,5 \%$ & $32,4 \%$ & $21,4 \%$ & $16,7 \%$ & $100,0 \%$ \\
\hline Estudios de secundaria & $26,8 \%$ & $31,2 \%$ & $21,1 \%$ & $20,9 \%$ & $100,0 \%$ \\
\hline Estudios de bachillerato o normal & $28,3 \%$ & $28,1 \%$ & $21,3 \%$ & $22,3 \%$ & $100,0 \%$ \\
\hline Estudios de licenciatura o más & $23,1 \%$ & $27,9 \%$ & $23,7 \%$ & $25,4 \%$ & $100,0 \%$ \\
\hline Total & $26,8 \%$ & $29,8 \%$ & $22,0 \%$ & $21,4 \%$ & $100,0 \%$ \\
\hline
\end{tabular}

Coincidiendo con lo que han mostrado otros estudio (Guzman, 2011; Miller, 2013), las trayectorias de estos alumnos se caracterizaron por no ser regulares. Sólo un poco más de una cuarta parte $(27 \%)$ terminó en el tiempo institucionalmente establecido como óptimo y a uno de cada tres les tomó hasta un año más. La mitad de los alumnos terminaron teniendo 24 años de edad o menos y tres de cada cuatro habían concluido antes de cumplir 27 años. A los 28 años prácticamente habían concluido todos (98\%).

Los datos anteriores nos hablan de la condición del grupo en su conjunto, sin embargo es pertinente hacer una distinción por año de ingreso, ya que a lo largo del periodo analizado se observa una tendencia creciente a mejorar 
el tiempo para concluir los estudios. Por ejemplo, el porcentaje de quienes terminaron en tiempo óptimo fue de 18.5 en 1990 y en 2005, de 30.9. Las edades con que concluyeron, por lo mismo, también tendieron a ser menores. Mientras que entre quienes ingresaron en 1990 sólo 16\% concluyeron con menos de 23 años, en los de 2005 la cifra se incrementó a 23\%. Estos datos nos hablan de una tendencia decreciente, si no lineal, sí continua, no asociada a programas institucionales. Es posible que esté relacionada con que los alumnos fueron crecientemente más jóvenes por cohorte de ingreso, con lo que aumentó la proporción de mujeres en la universidad y también el porcentaje de quienes tuvieron promedios de calificaciones altos en el bachillerato: mientras que en 1990, 43.3\% tuvieron un promedio de 8 o más, en 2005 éste llegó a 52.3\%.

Quienes ingresaron más jóvenes desertaron menos frecuentemente que los más grandes, aunque entre los que lograron mantenerse en el sistema hasta concluir sus estudios, el pequeño grupo de los que ingresaron siendo ya mayores de 30 años (4\%), terminó proporcionalmente en menos tiempo: $34.1 \%$ concluyeron en tiempo óptimo, mientras que entre los de 19 años, la cifra fue de 27.4.

En cuanto a las diferencias en los tiempos en los recorridos o trayectorias escolares hasta concluir, asociadas al género de los exalumnos, de acuerdo con lo esperado con base en otras investigaciones (Bartolucci, 2000; Mingo, 2007; Rodriguez Gómez, 1996), encontramos que las mujeres fueron más rápidas que sus compañeros varones: mientras que una de cada tres terminaron en el tiempo óptimo, entre los hombres sólo lo habían hecho uno de cada cinco, manteniéndose esta proporción de manera muy similar a lo largo de los noventa y el inicio del siglo xxI. Este hecho está asociado a que ellas ingresaron más frecuentemente siendo más jóvenes que los varones. Mientras que la mitad de los hombres tenían 19 años o menos al ingresar, entre las mujeres la proporción fue de $65 \%$. Pero aún en un mismo grupo de edad, ellas fueron más exitosas: $54.8 \%$ de las jóvenes de 19 años o menos concluyeron sus estudios; sólo $42.1 \%$ de los muchachos de ese grupo de edad lo hicieron.

Es posible imaginar que esta diferencia de resultados por género se deba a la socialización diferenciada a que han sido sometidos estos jóvenes. Es posible también pensar en que la observancia de las reglas y el cumplimiento de los requerimientos que hacen los profesores es más frecuente entre las mujeres como resultado de los roles sociales que tradicionalment les han sido asignados, y que esta actitud les ayude a aprobar los cursos más fácilmente que a sus compañeros, dado el carácter meritocrático del sistema de evaluación erigido sobre los resultados académicos. También podríamos pensar como posible hipótesis explicativa de estas diferencias de género, que los 
muchachos tienen más responsabilidades socioeconómicas familiares que las chicas y que esto afecte negativamente sus actividades escolares. En la cohorte de ingreso de 91-O de la que tenemos información sobre algunas condiciones sociales en el momento en que ingresaban a la Universidad, podemos ver que es claro que los muchachos se incorporaron más tempranamente que ellas al trabajo (UAM, 1991). Por último, Bartolucci (1994), Muñiz (1997) y Mingo ((2004) han planteado también la posibilidad de que sea la situación de desventaja social la que impulsa a las mujeres a hacer un esfuerzo mayor para sentirse competentes en ámbitos que pudieran parecer más propios de los hombres, y aunque la educación superior cada vez hace menos distinciones de género, para ellas pudiera ser que resulte necesario empeñarse más que sus compañeros. Es posible que un poco de cada una de estas explicaciones hayan incidido en estos resultados. Sin embargo, esta caracterización de la condición ventajosa no implica más que una probabilidad mayor, como ya lo señalamos antes.

Por otra parte, en esta investigación observamos también que hay condiciones institucionales que tienen un peso significativo en la factibilidad de terminar los estudios y de hacerlo en menos tiempo. En la población total analizada, más de cuatro de cada cinco alumnos de quienes concluyeron lo hicieron a más tardar un año después del óptimo en Xochimilco, mientras que en Azcapotzalco sólo uno de cada dos y en Iztapalapa uno de cada 3. Además, en Xochimilco los alumnos obtuvieron calificaciones más altas que en las otras dos Unidades.

Así pues, considerando los distintos resultados obtenidos hasta aquí, podemos decir que ser mujer, haber tenido una trayectoria regular en los estudios previos - de manera de poder iniciar los estudios de licenciatura con 19 años o menos - y haber tenido un buen promedio en el bachillerato, define un perfil más ventajoso para lograr terminar los estudios que ser hombre, con promedio del bachillerato bajo y que ingresó con más edad de la que exige una trayectoria regular. Observando las condiciones de los alumnos en cada una de las Unidades Académicas, el esquema se repite, aunque son menores las diferencias en Xochimilco.

\section{Inserción al mundo del trabajo}

En cuanto a la observación sobre la inserción al ámbito laboral, análisis que realizamos sólo sobre el grupo restringido del que tenemos información a partir del Estudio de Seguimiento de Egresados de la UAM, los datos mostra- 
ron que los jóvenes cuyos padres tenían menor escolaridad trabajaron más tiempo durante su carrera universitaria y por jornadas más prolongadas, aunque como lo mostrara Guzmán (2004), los motivos por los que lo hicieron responden a causas diversas y no necesariamente para el sustento familiar o propio. Además, trabajar o no trabajar no generó diferencias significativas en los promedios de calificaciones obtenidas. En cambio encontramos que un mejor origen social familiar sí incide en alcanzar condiciones laborales más ventajosas en términos económicos y de responsabilidad o nivel de mando, pero la diferencia con quienes tienen una familia con menores recursos no es perceptible sino después de cierto tiempo de estar trabajando: al comparar los datos de los ingresos percibidos en el primer trabajo contra los del último del que tuvimos conocimiento, observamos que, con el paso del tiempo, los hijos de padres con más escolaridad aumentaron sus ingresos en una proporción mayor que quienes provenían de una familia con pocos estudios.

Sin embargo, también en este punto del análisis es necesario señalar que la variable del origen social familiar no explica el resultado de las condiciones de trabajo del grueso del grupo. Para precisar lo que estamos diciendo, veamos por ejemplo el cuadro que aparece a continuación. Téngase en cuenta que la cohorte 1998 tiene ocho años de haber concluido en relación con el momento captado aquí por el Estudio de Seguimiento de Egresados de la UAM; la cohorte 2003 tiene sólo tres años; la de 2005 concluyó siete años atrás, y la de 2008, cuatro años antes. En cada una de ellas es evidente la ventaja de los hijos con alguno de sus padres con licenciatura, confirmando la incidencia de la variable. Pero entre familias con menor escolaridad, muchos logran esos mismos estándares, y en sentido inverso, muchos de los hijos de padres con licenciatura no están en el nivel más alto de ingresos.

Cuadro 3

Porcentaje de exalumnos que tenían más de 9 salarios mínimos en su último trabajo, por cohorte de egreso y por nivel de escolaridad de los padres

\begin{tabular}{|c|c|c|c|c|}
\hline \multirow{2}{*}{ Escolaridad de los padres } & Cohorte & más de 9 s. m. & Cohorte & más de 9 s. m. \\
\hline & 1998 & & 2005 & \\
\hline Hasta primaria & & $31 \%$ & & $18 \%$ \\
\hline Secundaria & & $39 \%$ & & $20 \%$ \\
\hline Bachillerato o normal & & $44 \%$ & & $28 \%$ \\
\hline Licenciatura & & $50 \%$ & & $32 \%$ \\
\hline Total & & $40 \%$ & & $25 \%$ \\
\hline
\end{tabular}




\begin{tabular}{|c|c|c|c|c|}
\hline \multirow{2}{*}{ Escolaridad de los padres } & Cohorte & más de $9 \mathrm{~s} . \mathrm{m}$. & Cohorte & más de $9 \mathrm{~s} . \mathrm{m}$. \\
\hline & 2003 & \multicolumn{3}{|c|}{2008} \\
\hline Hasta primaria & & $13 \%$ & & $10 \%$ \\
\hline Secundaria & & $14 \%$ & & $9 \%$ \\
\hline Bachillerato o normal & & $17 \%$ & & $14 \%$ \\
\hline Licenciatura & & $25 \%$ & & $18 \%$ \\
\hline Total & & $17 \%$ & & $13 \%$ \\
\hline
\end{tabular}
en 2012.

Por otra parte, este cuadro muestra también el deterioro en las condiciones salariales para este grupo, ya que las proporciones en que alcanzaron este nivel de ingresos se fueron reduciendo con el tiempo, tal como lo señalamos al hablar de la precarización salarial propia del contexto laboral para los profesionistas en los últimos treinta años.

En cuanto al efecto del logro académico, fue relevante encontrar que el ser un buen alumno no tuvo relación alguna con los logros laborales posteriores. Las buenas calificaciones escolares de los egresados no incidieron en tener mejores remuneraciones, ni en tener puestos con más responsabilidades o más vinculados con lo que estudiaron. Entre los componentes considerados para esta observación sobre logros académicos, sólo el de haber realizado y concluido estudios de posgrado generó una situación ventajosa; pero el número de casos no fue significativo, ya que implicó a no más del 10\% de los egresados. Este hallazgo coincide con los resultados encontrados por De Vries en nueve universidades mexicanas analizadas por él hace poco tiempo (Wietse De Vries, 2011).

El análisis de la trayectoria laboral previa mostró en cambio que haber trabajado antes va dando condiciones ventajosas respecto a quienes no vivieron tal experiencia, tanto en términos de ingresos como de responsabilidades o mando; aunque también en este cruce de variable vemos que hay un gran número de casos que tuvieron resultados contrarios a los esperados o en distintas posiciones del espectro de nuestra clasificación, por lo que nuevamente concluimos que esta variable no es suficientemente predictiva sobre el nivel del logro posible de los exalumnos estudiados. 
Cuadro 4

Trabajos previos de los egresados, por salarios mínimos en el último trabajo, 2006 o 2012

\begin{tabular}{llccccc}
\hline & \multicolumn{4}{c}{ Último trabajo 2006 o 2012 } \\
\cline { 3 - 6 } & & $\begin{array}{c}\text { Hasta 3 } \\
\text { s. m. }\end{array}$ & $\begin{array}{c}\text { Más de 3 } \\
\text { y hasta 6 } \\
\text { s. m. }\end{array}$ & $\begin{array}{c}\text { Más de 6 } \\
\text { y hasta 9 } \\
\text { s. m. }\end{array}$ & $\begin{array}{c}\text { Más de 9 } \\
\text { s. m. }\end{array}$ & Total \\
\hline \multirow{2}{*}{$\begin{array}{l}\text { Trabajos } \\
\text { previos }\end{array}$} & No trabajó antes & $13,9 \%$ & $34,5 \%$ & $23,1 \%$ & $28,6 \%$ & $100 \%$ \\
\cline { 2 - 6 } & Trabajó durante la licenciatura & $8,6 \%$ & $34,2 \%$ & $28,5 \%$ & $28,7 \%$ & $100 \%$ \\
\cline { 2 - 6 } & Empezó al concluir los estudios & $6,6 \%$ & $26,0 \%$ & $31,7 \%$ & $35,7 \%$ & $100 \%$ \\
\cline { 2 - 6 } & $\begin{array}{l}\text { Trabajó mientras estudiada y al } \\
\text { concluir }\end{array}$ & $5,1 \%$ & $21,0 \%$ & $31,7 \%$ & $42,2 \%$ & $100 \%$ \\
\hline Total & & $6,3 \%$ & $24,5 \%$ & $31,0 \%$ & $38,2 \%$ & $100 \%$ \\
\hline
\end{tabular}

En cuanto a las diferencias de resultados por género, los datos analizados mostraron lo que muchos otros estudios han señalado (Bartolucci, 2000; Mingo, 2007; Rodriguez Gómez, 1996; Valle Flores, 2011): que a ellas, independientemente del nivel de escolaridad de sus padres, les fue mejor académicamente que a los varones; pero que en el terreno laboral, su situación fue desventajosa, obteniendo salarios y responsabilidades siempre menores proporcionalmente que las de ellos. De Vries y Navarro (2009) Navarro Leal; Lladó, Dora y Sánchez, Luis (2009); Valdez Gardea; Ochoa y González (2011), muestran la desventaja en las remuneraciones de las mujeres aun con el mismo nivel educativo que sus colegas varones.

\section{Cuadro 5}

Concentrado de datos de tablas sobre Trabajos previos, Nivel de mando en el último trabajo, Coincidencia entre estudios y Trabajo, e Ingresos, por Sexo

\begin{tabular}{|c|c|c|c|c|c|}
\hline Sexo & & & $\begin{array}{c}\text { Puestos } \\
\text { de mando }\end{array}$ & $\begin{array}{c}\text { Total } \\
\text { coincidencia }\end{array}$ & $\begin{array}{c}\text { Más de } 9 \\
\text { s. m. }\end{array}$ \\
\hline \multirow[t]{5}{*}{ Femenino } & Trabajos previos & No trabajó antes & $8,7 \%$ & $56,4 \%$ & $8,6 \%$ \\
\hline & & Trabajó durante la licenciatura & $11,7 \%$ & $54,5 \%$ & $13,0 \%$ \\
\hline & & $\begin{array}{l}\text { Empezó a trabajar al concluir los } \\
\text { estudios }\end{array}$ & $0 \%$ & $54,8 \%$ & $21,0 \%$ \\
\hline & & $\begin{array}{l}\text { Trabajó mientras estudiaba y al } \\
\text { concluir }\end{array}$ & $18,0 \%$ & $53,5 \%$ & $23,6 \%$ \\
\hline & Total & & $16,0 \%$ & $54,3 \%$ & $20,1 \%$ \\
\hline \multirow[t]{5}{*}{ Masculino } & Trabajos previos & No trabajó antes & $14,8 \%$ & $57,9 \%$ & $17,7 \%$ \\
\hline & & Trabajó durante la licenciatura & $18,0 \%$ & $51,0 \%$ & $25,8 \%$ \\
\hline & & $\begin{array}{l}\text { Empezó a trabajar al concluir los } \\
\text { estudios }\end{array}$ & $22,0 \%$ & $54,4 \%$ & $36,8 \%$ \\
\hline & & $\begin{array}{l}\text { Trabajó mientras estudiaba y al } \\
\text { concluir }\end{array}$ & $26,5 \%$ & $55,7 \%$ & $42,6 \%$ \\
\hline & Total & & $24,0 \%$ & $54,9 \%$ & $37,7 \%$ \\
\hline
\end{tabular}


Una primera conclusión a la que podemos llegar con los datos anteriores es que la igualdad de oportunidades para hombres y mujeres es mayor en el ámbito de la educación superior que en el del mundo laboral, en razón de los criterios que predominan en cada uno de ellos para asignar recompensas. En el ámbito escolar es el mérito académico el que opera, y con éste, a ellas les va mejor. Hombres y mujeres son sometidos a las mismas pruebas y a los mismos retos, y los criterios de calificación se rigen por reglas impersonales, de competencia, en los términos de la racionalidad weberiana, que salvo en el examen de selección para el ingreso, han sido ventajosos para ellas. En cambio, en los espacios laborales, aunque el título profesional legitima la aspiración a ciertas ocupaciones y cargos porque supone capacidades, y en algunos campos incluso se torna en una criterio indispensable (piénsese por ejemplo que sólo quien tiene un título de abogado puede litigar), pierde fuerza la racionalidad meritocrática recuperando poder los factores discrecionales, las preferencias de quienes contratan. Por ahí aparecen entonces los criterios sexistas, clasistas, de discriminación hacia ciertos grupos etarios o ideológicos, entre otros, que en relación con las características y capacidades de los egresados de la educación superior, en una coyuntura particular del mercado de trabajo, en una región, en una rama de la actividad económica, dan forma a las desigualdades en las oportunidades laborales.

En el ámbito laboral en México no existe el mismo nivel de racionalidad instrumental. Es un ámbito más diverso que el de las instituciones educativas y las características que se consideran para elegir a un empleado y para valorar y recompensar las tareas realizadas, están menos reglamentadas. La condición de desventaja asociada a ser mujer se hace sentir con más fuerza que en el ámbito escolar.

Una prueba estadística de regresión lineal en la que consideramos escolaridad de los padres, promedio de calificaciones en la licenciatura, regularidad, edad, realización de trabajos previos y género confirmó lo antedicho: ninguna de las variables de manera aislada - pero tampoco reunidas - lograron ser suficientemente predictivas de los resultados.

\section{Conclusiones}

La conclusión a la que llegamos a partir de este análisis es que si bien tanto el origen social como la trayectoria laboral previa y el género pueden ser condiciones ventajosas para la población analizada en cuanto a concluir estudios o en relación con el ingreso obtenido por su trabajo, en cada uno de los casos, sus características y condiciones personales se tejen de forma particular afianzando o bien debilitando sus expectativas y posibilidades. Tal como ha 
sido señalado previamente, no hay factores determinantes (Bartolucci, 2000; Wietse De Vries, Velazquez-Cabrera y Ríos-Treto, 2013; Guzman, 1994). Hay tendencias en las posibilidades educativas y laborales en las que inciden factores coyunturales de la oferta educativa y del mercado laboral, características institucionales, así como las particularidades de los actores que en el campo interactúan.

Desde sus condiciones específicas, cada uno de estos jóvenes percibió sus posibilidades y desde allí decidió competir por un lugar en el insuficiente espacio de la oferta educativa de las universidades públicas de la zona, y se planteó continuar o no hasta finalizar los estudios; también fue desde ahí que se enfrentó a un mercado laboral en el que además es posible identificar cuotas y mecanismos de incorporación en función de criterios sobre el perfil deseable de los incorporables, según la rama económica o la empresa que contrata. El mercado de trabajo, a diferencia de la esfera escolar, tiene una normatividad más laxa. En la selección y las recompensas laborales, el mérito académico se subordina a criterios de los empleadores. El origen social (visto por ejemplo en relación con la institución educativa de la que egresa el profesionista) y las formas de valoración sobre el género recuperan la fuerza que, en el espacio escolar, se vieron disminuidas, para definir las oportunidades sociales.

Tener una licenciatura generó la expectativa sobre ciertos trabajos y condiciones laborales e hizo ver como inadecuado el acceso a otros que no se corresponden con el esfuerzo realizado. En el acceso al ámbito exclusivo de trabajos más estables, mejor remunerados y frecuentemente con cobertura en prestaciones sociales al que accedieron mayoritariamente los egresados, incidieron las particularidades de cada exalumno. Es el entramado particular de los distintos componentes, en un contexto de oportunidad específico, el que dio forma a las posibilidades educativas y laborales de este grupo.

Por otra parte, es también pertinente señalar como resultado de esta investigación que la posibilidad de profundizar sería mucho más rica si hubiera un esfuerzo institucional e interinstitucional para contar con información que permita tener un panorama más completo acerca de este tema a niveles nacional, regional y por sectores. Así mismo sería indispensable buscar la manera de hacer comparables los datos que las instituciones de educación superior obtienen sobre sus egresados, con los que genera INEGI, específicamente con los de la Encuesta Nacional de Ocupación y Empleo. En una coyuntura como la actual, en la que sigue habiendo una expectativa enorme sobre la educación como única vía de movilidad social, que choca contra un 
estrechamiento creciente del mercado laboral, es indispensable poder avanzar en el conocimiento de los problemas que aquí han sido señalados. Es fundamental tener más información sobre los alumnos que dejan inconclusos los estudios; ahondar en las características de condiciones de logro educativo de quienes continúan hasta el final, así como de las relativas a la vida laboral, a los factores que inciden en mejora de sus oportunidades.

Tabla 12

Análisis específico de las variables seleccionadas que han sido consideradas como factores que influyen en el proceso de deserción en la Escuela de Informática y Telecomunicaciones

\begin{tabular}{lcccc}
\hline & Estimate & Std. Error & $\mathrm{z}$ value & $\operatorname{Pr}(>|\mathrm{z}|)$ \\
\hline (Intercept) & -7.490315 & 8.635845 & -0.867 & 0.385749 \\
\hline DLENG & -0.015243 & 0.016741 & -0.911 & 0.362555 \\
\hline DMAT & -0.005440 & 0.013545 & -0.402 & 0.687945 \\
\hline CODIGO_AÑO_EGRESO & -0.055587 & 0.064931 & -0.856 & 0.391947 \\
\hline CODIGO_COMUNA & -0.004896 & 0.012397 & -0.395 & 0.692882 \\
\hline CODIGO_ESTABLECIMIENTO & 0.278548 & 0.981 & 0.326384 & 0.283820 \\
\hline CODIGO_GENERO & $\mathbf{- 2 . 0 4 6 8 1 6}$ & $\mathbf{0 . 5 8 6 7 0 2}$ & $\mathbf{- 3 . 4 8 9}$ & $\mathbf{0 . 0 0 0 4 8 5}$ \\
\hline CODIGO_JORNADA & -0.339439 & 0.580935 & -0.584 & 0.559019 \\
\hline PROM.EM & -0.143533 & 0.677712 & -0.212 & 0.832270 \\
\hline
\end{tabular}

Signif. codes: $0^{1 * * * 1} 0.001^{1 * * 1} 0.01^{* * 1} 0.05$ '.' $0.1^{\prime \prime} 1$

\section{Referencias}

Acosta, A. (2005). La educación superior privada en México. En D. O. F. H. E. I. L. A. A. T. C. IES/2005/ED/PI/47 (Ed.): IESALC-UnesCo.

Álvarez Mendiola, G. (2011). El fin de la bonanza. La educación superior privada en México en la primera década del siglo XXI. Reencuentro. UAM-Xochimilco, $60,10-29$.

ANUIES (2003). Mercado laboral de profesionistas en México. Desagregación regional y estatal de la información. Diagnóstico 1990-2000 (Vol. I, II, III y IV). México: ANUIES. ANUIES (2012). Mercado laboral de profesionistas en México. Diagnóstico 20002009 y prospectiva 2009-2015 y 2009-2020.

Bartolucci, J. (1994). La admisión escolar en la unAm. En R. y. C. R. Rodríguez, C (Ed.), Racionalidad política y vinculación social. México: CESU-UnAM.

Bartolucci, J. (2000). Desigualdad social, educación superior y sociología en México. CESU-UnAm y Editorial Porrúa.

Bernstein, B. (1989). Clases, códigos y control. II Hacia una teoría de las transmisiones educativas. Madrid: Akal. 
Boudon, R. (1983). La desigualdad de oportunidades. La movilidad social en las sociedades industriales. Barcelona: Edit. Laia.

Bourdieu, P. y Passeron, J. C. (1973). Los estudiantes y la cultura. Buenos Aires: Labor.

Bowles, S. y Gintis, H. (1983). La educación como escenario de las contradicciones en la reproducción de la relación capital-trabajo. Educación y Sociedad, 2, 7-23.

Coleman, J. S., Campbell, E. Q., Hobson, C. J., McPartland, F., Mood, A. M., Weinfeld, F. D. y et al. (1966). Equality of educational opportunity. Washington, D.C.: U.S. Government Printing Office.

Covo, M. (1995). Selección escolar y selección social: demanda de acceso y permanencia en la UNAM. En La universidad latinoamericana ante los nuevos escenarios de la región. México: udusL Universidad Iberoamericana.

De Garay, A. (2001). Los actores desconocidos, una aproximación al conocimiento de los estudiantes. México: ANUIES.

De Garay, A. (2011). La reactivación de la Educación Superior Pública y el papel de las instituciones Privadas en la Zona Metropolitana de la Ciudad de México en los primeros años del siglo xxI. Revista de la Educación Superior, ANUIES, $\mathrm{XL},(2)$, No. 158, 11-32.

De Garay, A. y Sánchez, L. (2012). La modificación de la política de admisión en la UAM y los cambios en las trayectorias escolares al primer alo de estudios en la Unidad Azcapotzalco. Perfiles Educativos. IISUe-Unam, 34, Núm. 135, 78-99.

De la Garza, E. y Gaspar, H. (2009). Modelos de producción y mercado de trabajo de los profesionistas en México.

De la Garza, M. (2015). La escolaridad en la determinación de los ingresos en la década de 1990. Doctorado, Universidad de Barcelona.

De los Santos, E. (2004). Los procesos de permanencia y abandono escolar en educación superior. Revista Iberoamericana de Educación Superior, 33, 2.

De Vries, W. (2011). ¿Desertores o decepcionados? Distintas causas para abandonar los estudios universitarios. Revista de la Educación Superior, 40, Núm 160.

De Vries, W., Velazquez-Cabrera, R. y Ríos-Treto, D. (2013). Millonarios o malparados: ¿de qué depende el éxito de los egresados universitarios? Revista Iberoamericana de Educación Superior. IISUE, IV, Num 9, 3-20.

Fernandez Mellizo-Soto (2003). Igualdad de oportunidades Educativas: la Experiencia socialdemócrata Española y Francesa. Madrid: Pomares Corredor.

Fuentes, O. (1983). Las épocas de la universidad mexicana. Cuadernos Políticos, ERA, 36.

García Castro, G. y Bartolucci, J. (2007). Aspiraciones educativas y logro académico. Un estudio de caso sobre característicasy condiciones sociales de los estudiantes de la UAM. REMIE, 12, Num 35, 1267-1288.

Granja, J. (1983). Análisis sobre las posibilidades de permanencia y egreso en cuatro instituciones de Educación Superior en el D.F., 1960-1978. Revista de la Educación Superior, 123 (47), 5-35.

Guzman, C. (1994). Entre el deseo y la oportunidad: estudiantes de la UNAM frente al mercado de trabajo. México: CRIM-UnAm. 
Guzman, C. (2004). Entre el estudio y el trabajo. La situación y las búsquedas de los estudiantes de la UNAM que trabajan. México: CRIM-UNAM.

Guzman, C. (2011). Avances y retos en el conocimiento sobre los estudiantes mexicanos de educación superior en la primera década del siglo XxI. Perfiles Educativos. IISUE, UNAM, Vol xxXIII, número especial 2011, 91-101.

Guzman, C. y Serrano, O. V. (2011). Las puesrtas del ingreso a la educación superior: el caso del concurso de selección a la licenciatura de la UNAM. Revista de la Educación Superior, 40, núm 157.

Guzmán, C. y Serrano, O. V. (2007). Los cambios en la composición social de los estudiantes de la unAm (1985-2003). En A. Mingo (Ed.), Estudiantes universitarios: cinco acercamientos (pp. 164-208). México: UNAM-IISUE.

Hernández Laos, E. (2004). Panorama del mercado laboral de profesionistas en México. Economía UnAM, Vol. 1 No 2.

Hernández Laos, E. y al, e. (2012). Mercado laboral de los profesionistas en México: diagnóstico 2000-2009 y prospectiva 2010-2020 Informe Final. México: ANUIES.

López Zárate, R., González Cuevas, M. y Casillas Alvarado, M. (2000). Una historia de la UАM. Sus primeros 25 años, México, Universidad Autónoma Metropolitana (Vol. 2). México: UAM.

Miller, D. (2013). El estudio de las trayectorias escolares en México: un aporte para el nuevo milenio. En C. L. Saucedo Ramos, C. Guzman, E. Sandoval \& J. F. Galaz (Eds.), Estudiantes, maestros y académicos en la investigación educativa. Tendencias, aportes y debates 2002-2011. México: COMIE-ANUIES.

Mingo, A. (2007). ¿Quien mordió la manzana? Sexo, orígen social y desempeño en la universidad. México: FCE.

Muñiz Martelón, P. (1997). Origen social, trayectorias educativas y deserción universitaria en los ochenta. Tesis para obtener el grado de Doctor en Ciencias Sociales con especialidad en Estudios de Población, Colegio de México., México.

OCDE (2010). Panorama de la Educación. Indicators at Glance, from www.oecd.org/ dataoecd/46/20/45925316.pdf

Rodriguez Gómez, R. (1996). Educación Superior y desigualdad social. Un estudio sobre las determinaciones sociales y académicas de las trayectorias escolares en la Universidad Nacional Autónoma de México. Doctorado, Colegio de Mexico, Ciudad de México.

Sanchez Medina, R. (2010). El efecto del ambiente institucional y organizacional sobre las trayectorias escolares en el primer año de estudios. El caso de la UAM-Azcapotzalco. Tesis de Maestría, uAm-Azcapotzalco, México.

Schütz, A. (1962). El problema de la realidad social. Buenos Aires: Amorrortu editores.

Silva Laya, M. (2014). Equidad en la Educación Superior Mexicana:el reto persiste. Universidades, LXv 59, 23-35.

Tinto, V. (1989). La deserción en la educación superior: Síntesis de las bases teóricas de las investigaciones recientes. En La trayectoria escolar de la Educación Superior. Proides-ANUIES.

uAM (1991). Base de Datos del Cuestionario Socioeconómico para aspirantes 91-O. Departamento de Estudios Escolares. UAM. 
UAM (2006). Base de datos del Estudios de Seguimiento de Egresados Generaciones 1998-2003. En E. y. E. S. Coordinación General de Información Institucional. Sistema de Información de Estudiantes (Ed.).

UAM (2011). Anuario estadístico 2011. Datos históricos sobre demanda y admisión en uAm. uAm: Dpto. de Admisión de la Coordinación General de Información Institucional. Dirección de Planeación.

UAM (2013). Base de datos del Estudios de Seguimiento de Egresados Generaciones 2005-2008. En E. y. E. S. Coordinación General de Información Institucional. Sistema de Información de Estudiantes, UAM. (Ed.).

UAM (2015). Informe anual del Rector General 2015. Anuario Estadístico. México: UAM.

Valle Flores, A. (2011). Panorama general 1970 al 2005 de la matrícula y el egreso universitario en México como indicadores de la tensión en el mercado laboral. Cuestiones de Sociología, Universidad Nacional de la Plata. Facultad de Humanidades y Ciencias de la Educación. Depto. de sociología, 7. 